\title{
CONVERGENT PROCESSES, PROJECTIVE SYSTEMS OF MEASURES AND MARTINGALE DECOMPOSITIONS
}

\author{
by LOUIS H. BLAKE
}

(Received 31 October, 1977)

Introduction. The purpose of this paper is to show the equivalence of convergence, an associated projective system of measures and a martingale decomposition for a uniformly integrable stochastic process. Emphasis is placed on a direct juxtaposition of these concepts and on displaying underlying mechanisms.

The impact of the martingale convergence theorem on contemporary probability theory has been immense. Therein lies the reason for numerous generalizations of both the basic martingale convergence theorem and the martingale concept itself.

Among the noteworthy generalizations of the martingale theorem are those of Krickeberg [1] and Chatterji [2] and Darst [3].

This paper, however, concerns itself with a relatively new endeavor: generalizations of the martingale concept.

A first effort in the direction of martingale generalizations came in 1965 in a paper by Fisk [4], which introduced "quasi-martingales". In 1970 and 1972 basic papers by Blake [5], [6] introduced the concept of "games which become fairer with time". In 1973 two noteworthy reproofs of the $L_{1}$ convergence theorem for games which become fairer with time appeared. See [7] and [13]. Subsequently papers by Mucci [7], [8] obtained results for a specialized version of games fairer with time which are called "martingales in the limit". In 1974 a paper by Austin, Edgar and Tulcea [9] introduced a study of "asymptotic martingales".

In most of the efforts in martingale generalizations the new processes have convergence properties. In the course of development of these processes certain concepts have reappeared. First, the concept of projective systems of measures occurs in the work of Blake [6] and subsequently in Edgar and Sucheston [10]. (It should be observed that the concept of projective systems of measures in the context of martingales goes back to Baez-Duarte [11] and is brought to its full state of generalization by Lamb [12].) Second, the concept of martingale decomposition has occurred for these generalizations. Its first occurrence is in Blake [6] where it is the key tool in establishing an $L_{1}$ convergence theorem.

Most of the martingale generalizations have convergence theorems associated with them. It is the intent of this paper to show that under conditions of uniform integrability processes which converge, projective systems of measures and martingale decompositions are all equivalent concepts. The results are presented in such a way as to expose the direct connection between these concepts and to expose wherever possible the underlying mechanisms.

The main results. Let $(\Omega, \mathfrak{U}, \mu)$ be a probability space. Let $\left\{\mathfrak{U}_{n}\right\}_{n \geq 1}$ be an increasing sequence of sub sigma algebras generating $\mathfrak{U}$. Let $\left\{f_{n}\right\}_{n \geq 1}$ be adapted to $\left\{\mathfrak{U}_{n}\right\}_{n \geq 1}$.

Glasgow Math. J. 20 (1979) 119-124. 
DEFINTIION. Let $\left\{\mu_{n}\right\}_{n \geq 1}$ be a sequence of probability measures where $\mu_{n}$ is defined on $\mathfrak{A}_{n} .\left\{\mu_{n}\right\}_{n \geq 1}$ is called a projective system of probability measures with respect to $\mu$ if $\mu$ is the uniform limit of $\left\{\mu_{n}\right\}_{n \geq 1}$ in the sense that for every $\varepsilon>0$ there exists an $N$ such that

$$
\sup _{A \in \mathscr{Q}_{m}}\left|\mu_{n}(A)-\mu_{m}(A)\right|<\varepsilon
$$

for every $n, m \geq N$.

DEFINTIION. The sequence $\left\{f_{n}\right\}_{n \geq 1}$ is uniformly integrable with respect to the projective system $\left\{\mu_{n}\right\}_{n \geq 1}$ if

(i) for every $\varepsilon>0$ there exists a $\delta>0$ and an $N$ such that for all $n \geq N, \int_{A}\left|f_{n}\right| d \mu_{n}<\varepsilon$ whenever $A \in \mathfrak{A}_{n}$ and $\mu_{n}(A)<\delta$, and

(ii) $\sup _{n=1}^{\infty} \int\left|f_{n}\right| d \mu_{n}<\infty$.

REMARK. The previous definition reduces to the usual case of uniform integrability when $\mu_{n}$ is the restriction of $\mu$ to $\mathfrak{A}_{n}$. In the general setting of $\left\{\mu_{n}\right\}_{n \geq 1}$ being an arbitrary projective system the concept of $\left\{f_{n}\right\}_{n \geq 1}$ being uniformly integrable with respect to $\left\{\mu_{n}\right\}_{n \geq 1}$ will prove to be a desirous (technical) condition.

For the remainder of the paper let $\left\{f_{n}\right\}_{n \geq 1}$ be uniformly integrable with respect to $\left\{\mu_{n}\right\}$ as well as $\mu$ and let $\left\{\mu_{n}\right\}_{n \geq 1}$ be a projective system of probability measures.

DefINITION. $\left\{f_{n}\right\}_{n \geq 1}$ has a martingale decomposition if $f_{n}=Y_{n}+Z_{n}$ for each $n$, where $\left\{Y_{n}\right\}_{n \geq 1}$ is a martingale and $\left\{Z_{n}\right\}_{n \geq 1}$ converges (a.e. or in measure or in $L_{1}$ ) to zero.

REMARK. It is clear from a basic Chebychev argument that for every $\varepsilon>0$ there exists positive integers $M$ and $N$ such that

(i) $\mu\left(\left|f_{n}\right|>M\right) \leq \varepsilon$ for all $n \geq N$,

(ii) $\mu_{n}\left(\left|f_{n}\right|>M\right) \leq \varepsilon$ for all $n \geq N$.

PROPOSTrion 1. For every $\varepsilon>0$ there exists an $N$ such that for any $n \geq N$ and any $A \in \mathfrak{A}_{n}$

$$
\left|\int_{A} f_{n} d \mu_{n}-\int_{A} f_{n} d \mu\right|<\varepsilon
$$

Proof. From previous properties it is clear that there exist, for $\varepsilon>0, M$ and $N$, such that for $n \geq N$,

$$
\int_{\left\{\left|f_{n}\right|>M\right\}}\left|f_{n}\right| d \mu_{n}<\varepsilon
$$

and

$$
\int_{\left\{\left|f_{n}\right|>M\right\}}\left|f_{n}\right| d \mu<\varepsilon
$$

Hence, consider $\left|f_{n}\right| \leq M$ for all $N$ (this is done to ease the burdensome notation $f_{n}^{M}$ as the truncation of $f_{n}$ at $M$ ) and also consider $f_{n} \geq 0$ for all $n$ (in order to consider $\left(f_{n}^{M}\right)^{+}$, the 
positive part of $f_{n}^{M}$, and prove the result for $\left(f_{n}^{M}\right)^{+}$. A similar approach is taken for $\left(f_{n}^{M}\right)^{-}$ and the proposition is proved.)

For each $f_{n}$ there exists a sequence of simple functions $\left\{S_{p, n}\right\}_{p \geq 1}$ with $S_{p, n} \uparrow f_{n}$ and $0 \leq S_{p, n} \leq M$ for all $p$ and $S_{p, n} \mathfrak{A}_{n}$-measureable for each $p$. Consider,

For some $p$ write

$$
\int_{A} S_{p, n} d \mu_{n}-\int_{A} S_{p, n} d \mu
$$

$$
S_{\mathrm{p}, \mathrm{n}}=c_{1} I_{\mathrm{A}_{1}}+\ldots+c_{k} I_{\mathrm{A}_{k}}
$$

where $M \geq c_{i} \geq 0$ for all $i$ and the $A_{i}$ 's are disjoint. Then, (1) is simply

It then follows that

$$
\sum_{i=1}^{k} c_{i}\left(\mu_{n}-\mu\right)\left(A_{i} A\right)
$$

$$
M \sum_{q=1}^{r}\left(\mu_{n}-\mu\right)\left(A_{i_{i}} A\right) \leq \sum_{i=1}^{k} c_{i}\left(\mu_{n}-\mu\right)\left(A_{i} A\right) \leq M \sum_{j=1}^{i}\left(\mu_{n}-\mu\right)\left(A_{i_{i}} A\right)
$$

where $\left(\mu_{n}-\mu\right)\left(A_{i_{i}} A\right)<0$ and $\left(\mu_{n}-\mu\right)\left(A_{i} A\right)>0$. Suppose, without loss of generality, that

$$
\sum_{j=1}^{1}\left(\mu_{n}-\mu\right)\left(A_{i_{i}} A\right) \geq\left|\sum_{a=1}^{r}\left(\mu_{n}-\mu\right)\left(A_{i_{a}} A\right)\right| .
$$

Then (2) is rewritten as:

$$
-M \sum_{j=1}^{t}\left(\mu_{n}-\mu\right)\left(A_{i} A\right) \leq \sum_{i=1}^{k} c_{i}\left(\mu_{n}-\mu\right)\left(A_{i} A\right) \leq M \sum_{j=1}^{t}\left(\mu_{n}-\mu\right)\left(A_{i_{i}} A\right) .
$$

Thus, choose $N_{2}$ sufficiently large that for all $n \geq N_{2},\left|\mu_{n}(A)-\mu(A)\right|<\varepsilon / M$ for all $A \in \mathfrak{A}_{n}$. Thus, for all $n \geq N_{2} \geq N$ and $A \in \mathfrak{U}_{n}$

$$
\left|\int_{A} S_{p, n} d \mu_{n}-\int_{A} S_{p, n} d \mu\right|<\varepsilon
$$

for all $p$. Thus, as $p \rightarrow \infty$, the result follows.

REMARK. The previous proposition is immediate in the case where $\mu_{n}$ is simply the restriction of $\mu$ to $\mathfrak{A}_{n}$.

DEFINTION. For the process $\left\{f_{n}\right\}_{n \geq 1}$, its associated family of measures is given by $\left\{\phi_{n}\right\}$ where $\phi_{n}(A)=\int_{A} f_{n} d \mu_{n}$ for each $A \in \mathfrak{A}_{n}$.

THEOREM 2. The sequence $\left\{f_{n}\right\}_{n \geq 1}$ is a game fairer with time if and only if its associated family of measures is a projective system of measures.

Proof. Suppose $\left\{f_{n}\right\}_{n \geq 1}$ converges $L_{1}$. Then with Proposition 1 and the appropriate triangle inequalities it is immediate that the associated family of measures $\left\{\phi_{n}\right\}_{n \geq 1}$ is a projective system. 
Next suppose the associated family of measures $\left\{\phi_{n}\right\}_{n \geq 1}$ is a projective system. Consider the sets $A_{m, n} \equiv\left\{E\left(f_{n} \mid \mathfrak{A}_{m}\right)-f_{m} \geq 0\right\}$ and write

$$
\begin{aligned}
\left|\phi_{n}\left(A_{m, n}\right)-\phi_{m}\left(A_{m, n}\right)\right|+ & \left|\int_{A_{m, n}} f_{n} d \mu-\int_{A_{m, n}} f_{n} d \mu_{n}\right|+\left|\int_{A_{m, n}} f_{m} d \mu-\int_{A_{m, n}} f_{m} d \mu_{m}\right| \\
& \geq\left|\int_{A_{m, n}} f_{n} d \mu-\int_{A_{m, n}} f_{m} d \mu\right| \\
& =\int_{A_{m, n}}\left|E\left(f_{n} \mid \mathfrak{A}_{m}\right)-f_{m}\right| d \mu \\
& \geq \varepsilon \mu\left(A_{m, n}\left|E\left(f_{n} \mid \mathfrak{A}_{m}\right)-f_{m}\right|>\varepsilon\right) .
\end{aligned}
$$

This chain of inequalities also holds for the sets $\sim A_{m, n}$. Since $\left\{\phi_{n}\right\}_{n \geq 1}$ is a projective system and with Proposition 1 it follows that $\left\{f_{n}\right\}_{n \geq 1}$ is a game fairer with time. (For the definition of a game fairer with time and the basic $L_{1}$ convergence theorem see [6], [7], [13]).

With the aid of the basic $L_{1}$ convergence theorem for uniformly integrable games fairer with time, the connection between projective systems and $L_{1}$ convergence can be made.

THEOREM 3. The sequence $\left\{f_{n}\right\}_{n \geq 1}$ converges in $L_{1}$ if and only if its associated family of measures is a projective system of measures.

Proof. $\left\{f_{n}\right\}_{n \geq 1}$ is fairer with time if and only if $\left\{f_{n}\right\}_{n \geq 1}$ converges in $L_{1}$.

THEOREM 4. The associated family of measures for $\left\{f_{n}\right\}_{n \geq 1}$ is a projective system if and only if $\left\{f_{n}\right\}_{n \geq 1}$ has a martingale decomposition where $\left\{Y_{n}\right\}_{n \geq 1}$ converges $L_{1}$ and $\left\{Z_{n}\right\}_{n \geq 1}$ converges to zero $L_{1}$.

Proof. The if part of the theorem is obvious.

If the associated family is a projective system then $\left\{f_{n}\right\}_{n \geq 1}$ converges in $L_{1}$. To establish this begin by defining

$$
p_{n}(A)=\left.\int_{A} f_{n} d \mu\right|_{\mathfrak{r}_{m}}=\int_{A} f_{n} d \mu .
$$

$\left\{p_{n}\right\}_{n \geq 1}$ is by hypothesis a projective system of measures from which $\left\{\nu_{n}\right\}_{n \geq 1}$ is defined by

$$
\nu_{n}(A) \equiv \lim _{\substack{m \rightarrow \infty \\ m \geq n}} p_{m}(A)
$$

It follows that for each $n, \nu_{n}$ is defined on $\mathfrak{A}_{n}$ and for any $n>m \nu_{n}=\nu_{m}$ on $\mathfrak{A}_{m}$. Also $\nu_{n} \ll \mu$ for each $m$. Thus a martingale process $\left\{Y_{n}\right\}_{n \geq 1}$ exists such that

$$
\nu_{n}(A)=\int_{A} Y_{n} d \mu \quad\left(A \in \mathfrak{A}_{n}\right)
$$


First, $\left\{Y_{n}\right\}_{n \geq 1}$ is uniformly integrable. It is sufficient to show

$$
\lim _{t \rightarrow \infty} \int_{\left\{\left|Y_{n}\right|>t\right\}} Y_{n}=0
$$

uniformly in $n$. Let $D_{n}$ and $N_{n}$ be the positive set and negative set respectively for $Y_{n}$ and write

$$
\begin{aligned}
& 0 \leq \nu_{n}\left(D_{n}\right)=\lim _{\substack{p \rightarrow \infty \\
p \geq n}} \int_{D_{n}} f_{n} \leq \sup _{n}\left|f_{n}\right| \\
& 0 \leq \nu_{n}\left(N_{n}\right) \leq \ldots \leq \sup _{n} \int\left|f_{n}\right|
\end{aligned}
$$

Thus, $\sup _{n} \int\left|Y_{n}\right|=M<\infty$. It then follows that $t P\left(\left|Y_{n}\right|>t\right)<M$; so $P\left(\left|Y_{n}\right|>t\right)$ gets small uniformly in $n$. Then,

$$
\begin{aligned}
& \lim _{t \rightarrow \infty} \int_{\left(\left|Y_{n}\right|>t\right) D_{n}} Y_{n}=\lim _{t \rightarrow \infty} \lim _{\substack{p \rightarrow \infty \\
p>n}} \int_{\left(\left|Y_{n}\right|>t\right) D_{n}} f_{p}, \\
& \lim _{t \rightarrow \infty} \int_{\left(\left|Y_{n}\right|>t\right) N_{n}} Y_{n}=\lim _{t \rightarrow \infty} \lim _{\substack{p \rightarrow \infty \\
p \geq n}} \int_{\left(\left|Y_{n}\right|>t\right) N_{n}} f_{p}
\end{aligned}
$$

and it follows that $\lim _{t \rightarrow \infty} \int_{\left(\left|Y_{n}\right|>t\right)} Y_{n}=0$ uniformly in $n$ inasmuch as $\left\{f_{n}\right\}_{n \geq 1}$ is uniformly integrable.

From Theorem $3\left\{f_{n}\right\}_{n \geq 1}$ converges in $L_{1}$. The proof will be complete by showing that $\left\{f_{n}-Y_{n}\right\}_{n \geq 1}$ converges to zero in $L_{1}$. It will be sufficient to show that for any sequence of sets $\left\{A_{n}\right\}_{n \geq 1}$ with $A_{n} \in \mathfrak{A}_{n}, \lim _{n \rightarrow \infty}\left|\nu_{n}\left(A_{n}\right)-p_{n}\left(A_{n}\right)\right|=0$, for if this is so merely write

$$
\int\left|f_{n}-Y_{n}\right| \leq\left|p_{n}\left(D_{n}\right)-\nu_{n}\left(D_{n}\right)\right|+\left|p_{n}\left(N_{n}\right)-\nu_{n}\left(N_{n}\right)\right|
$$

where $N_{n} \equiv\left\{f_{n}-Y_{n}<0\right\}, D_{n} \equiv\left\{f_{n}-Y_{n} \geq 0\right\}$. Now, for $\varepsilon$ and $n$ given, select $k_{n, \varepsilon}>n$ such that

$$
\left|\nu_{n}\left(A_{n}\right)-p_{k_{n, \varepsilon}}\left(A_{n}\right)\right|<\varepsilon / 2
$$

and

$$
\left|p_{n}\left(A_{n}\right)-p_{k_{n \cdot e}}\left(A_{n}\right)\right|<\varepsilon / 2 .
$$

As an immediate consequence of the previous two theorems, it follows that

(i) a uniformly integrable family $\left\{f_{n}\right\}_{n \geq 1}$ converges $L_{1}$ if and only if it has a martingale decomposition

(ii) the associated family of measures for the uniformly integrable family is a projective system if and only if $\left\{f_{n}\right\}_{n \geq 1}$ has a martingale decomposition. 


\section{REFERENCES}

1. K. Krickeberg, Convergence of martingales with a directed index set, Trans. Amer. Math. Soc. 83 (1956), 313-337.

2. S. D. Chatterji, Martingale convergence and the Radon-Nikodym theorem in Banach spaces, Math. Scand. 22 (1968), 21-41.

3. R. B. Darst, Convergence of Radon-Nikodym derivatives and martingales given sigma lattices, Illinois J. Math. 21 (1977), 113-123.

4. D. L. Fisk, Quasi-martingales, Trans. Amer. Math. Soc., 120 (1965), 369-389.

5. L. H. Blake, A generalization of martingales and two consequent convergence theorems, Pacific J. Math. 35 (1970), 279-283.

6. L. H. Blake, A note concerning the $L_{1}$ convergence of a class of games which become fairer with time, Glasgow Math. J. 13 (1972), 39-41.

7. A. G. Mucci, Limits for martingale-like sequences, Pacific J. Math. 48 (1973), 197-203. 539-541.

8. A. G. Mucci, Another martingale convergence theorem, Pacific J. Math. 64 (1976),

9. D. G. Austin, G. A. Edgar and A. Ionesca Tulcea, Pointwise convergence in terms of expectations, Zeit. fur Wahr. 30 (1974), 17-26.

10. G. A. Edgar and L. Sucheston, The Riesz decomposition for vector-valued amarts, Zeit. fur Wahr. 36 (1976), 85-92.

11. L. Baez-Duarte, Another look at the martingale theorem, J. Math. Anal. Appl. 23 (1968), 551-557.

12. C. W. Lamb, A ratio limit theorem for approximate martingales, Canad. J. Math. 25 (1973), 772-779.

13. R. Subramanian, On a generalization of martingales due to Blake, Pacific J. Math. 48 (1973), 275-278.

\section{Department of Mathematics}

College of Staten Island

CUNY

STATEN IsLAND

NEW YORK 10301. 